\title{
ВОЗМОЖНОСТИ ИСПОЛЬЗОВАНИЯ КОМПЬЮТЕРНЫХ МОДЕЛЕЙ ДЛЯ СНИЖЕНИЯ РИСКОВ ПРИ ВРЕМЕННОМ ПРОТЕЗИРОВАНИИ
}

Н. В. Багрянцева ${ }^{1,2 \square}$ С. И. Гажва, А. А. Баранов², Л. Б. Шубин², В. А. Багрянцев², О. В. Багрянцева²

1 Приволжский исследовательский медицинский университет, Нижний Новгород, Россия

2 Ярославский государственный медицинский университет, Ярославль, Россия

Современная ортопедическая стоматология обладает большим арсеналом решений для оказания помощи пациентам с полной вторичной адентией, что, однако, может вызывать ряд проблем. При выборе вида временного протеза на момент остеоинтеграции дентальных имплантатов необходимо учитывать большое количество факторов, возникающих при различных клинических ситуациях. Целью работы было в ходе ретроспективного анализа медицинской документации пациентов с полной вторичной адентией и установленными временными протезами (покрывными съемными и условносъемными) на момент остеоинтеграции дентальных имплантатов оценить факторы риска и отношение шансов их реализации в развитии осложнений на этот период. Осуществлено многомерное математико-статистическое моделирование с построением компьютерного алгоритма принятия решений в выборе типа протеза и материала для его изготовления. Алгоритм (модель) обладает высокими значениями чувствительности и специфичности 94,37 (76,2 : 98,7) и 92,56 (79,8 : 97,6), при площади под характеристической кривой, равной 0,921 (0,843 : 0,963). Используя автоматизацию поэтапного алгоритма, планируется создать компьютерную программу для повышения степени объективности при выборе способа временного протезирования и материала протеза.

Ключевые слова: дентальная имплантация, остеоинтеграция, временное протезирование, риски, шансы, компьютерная модель

Информация о вкладе авторов: Н. В. Багрянцева - разработка дизайна исследования, получение данных, анализ и интерпретация данных, редактирование рукописи; С. И. Гажва - планирование исследования, редактирование рукописи; А. А. Баранов - редактирование рукописи; Л. Б. Шубин - анализ и интерпретация данных; В. А. Багрянцев, О. В. Багрянцева - сбор данных, подготовка черновика рукописи.

Соблюдение этических стандартов: исследование одобрено этическим комитетом ФГБОУ ВО НижГМА Минздрава России (протокол № 10 от 25 декабря 2017 г.).

$凶$ Для корреспонденции: Наталья Владимировна Багрянцева ул. 8 марта, д. 1, кор. 2, кв. 71, г. Ярославль, 150002; nbogryanceva@mail.ru

Статья получена: 25.07.2019 Статья принята к печати: 09.08.2019 Опубликована онлайн: 16.08.2019

DOI: $10.24075 /$ vrgmu.2019.050

\section{THE FEASIBILITY OF USING COMPUTER-BASED MODELS FOR REDUCING THE RISKS OF COMPLICATIONS ASSOCIATED WITH TEMPORARY DENTURES}

Bagryantseva $\mathrm{NV}^{1,2} \otimes$, Gazhva SI ${ }^{1}$, Baranov $\mathrm{AA}^{2}$, Shubin $\mathrm{LB}^{2}$, Bagryantsev $\mathrm{VA}^{2}$, Bagryantseva $\mathrm{OV}^{2}$

${ }^{1}$ Privolzhsky Research Medical University, Nizhny Novgorod, Russia

2 Yaroslavl State Medical University, Yaroslavl, Russia

Contemporary prosthetic dentistry has a vast arsenal of solutions for completely edentulous patients. However, it is crucial to consider a variety of factors that can cause complications in patients wearing temporary dentures in the osseointegration period. The aim of this study was to retrospectively analyze the medical records of completely edentulous patients wearing temporary removable or fixed dentures in the osseointegration period, to identify the risk factors for complications and to calculate the odds of adverse events. We performed a multivariate analysis and developed a computerized algorithm that could be used to facilitate selection of the proper denture type and material. The algorithm demonstrates high sensitivity and specificity: 94.37 (76.2 : 98.7$)$ and 92.56 (79.8 : 97.6$)$, respectively; the AUC value is $0.921(0.843: 0.963)$. We are planning to develop a software based on the proposed algorithm that would help the dentist to make a more objective decision when selecting the type of temporary denture and its material.

Keywords: dental implants, osseointegration, temporary prosthesis, risks, odds, computer-based model

Author contribution: Bagryantseva NV — study design, data acquisition, analysis and interpretation, manuscript editing; Gazhva SI — study planning and manuscript editing; Baranov AA — manuscript editing; Shubin LB — data analysis and interpretation; Bagryantsev VA, Bagryantseva OV — data acquisition and preparing the manuscript draft.

Compliance with ethical standards: the study was approved by the Ethics Committee of Privolzhsky Research Medical University (Protocol № 10 dated December 25, 2017).

$\triangle$ Correspondence should be addressed: Natalia V. Bagryantseva

March 8, d. 1, kor. 2, kv. 71, Yaroslavl, 150002; nbogryanceva@mail.ru

Received: 25.07.2019 Accepted: 09.08.2019 Published online: 16.08.2019

DOI: 10.24075/brsmu.2019.050

На сегодняшний день операции с использованием имплантатов распространены во всем мире и позволяют получать хорошие долгосрочные результаты [1, 2]. Стоматологи могут прогнозировать окончательный результат еще до лечения. После проведения дентальной имплантации на всех этапах лечения оправдано использование протезов временной конструкции. Для временного протезирования могут быть использовань съемные и условно-съемные конструкции протезов из различных материалов [3].

Вместе с тем подходы к тактике временного зубного протезирования на момент остеоинтеграции после установки дентальных имплантатов при ведении пациентов с полным отсутствием зубов не однозначны [4]. Анализ имеющейся литературы показал, что необходимо уделить особое внимание адекватному построению алгоритма выбора конструкции 
и разработать математически выверенный подход к реабилитации пациентов с полным отсутствием зубов [2]. Это позволит снизить риски неблагоприятных исходов и осложнений, способствуя повышению качества жизни пациентов.

\section{ПАЦИЕНТЫ И МЕТОДЫ}

Был проведен ретроспективный анализ медицинской документации ГБУЗ ЯО «Ярославская областная стоматологическая поликлиника» г. Ярославля. Изучали данные медицинских карт стоматологического больного (форма 043/y) и дневник учета работы стоматологаортопеда (форма N 039-4/y) за период 2015-2019 гг. Критерии включения пациентов в исследование: пациенты любого пола и возраста; наличие у пациента вторичной адентии. Критерии исключения: наличие экзостозов, онкологических заболеваний, нарушений свертываемости крови; наличие соматической патологии в стадии декомпенсации. При отборе учитывали обстоятельства, при которых были потеряны зубы, наличие жалоб, план лечения и вид временного протеза, выбранный на момент остеоинтеграции дентальных имплантатов. Отдельно учитывали материал, из которого изготовлен протез. Результаты регистрировали в многовходовых таблицах с последующий кодировкой. Всего было изучено 102 медицинских карты и 1 дневник работы стоматологаортопеда. У всех 102 пациентов была диагностирована полная или частичная вторичная адентия. Из них у 34 пациентов зубы потеряны полностью, а у 68 пациентов еще оставались подвижные зубы или корни разрушенных зубов, которые впоследствии удаляли, и пациентов включали в группу с диагнозом «полная вторичная адентия». Установлено, что всем 102 пациентам было проведено ортопедическое лечение с применением дентальных имплантатов и последующим временным протезированием. Учитывая цель работы, пациенты были разделены на две группы: в первую вошли те, у кого протезирование прошло успешно, а во вторую включили пациентов, у которых развились те или иные осложнения на момент остеоинтеграции дентальных имплантатов. Численность групп составила 73 и 29 человек соответственно. Всем пациентам трижды проводили рентгенологическое исследование на ортопантомографе Strato 2000d (Villa Sistemi Medicali; Италия) для оценки состояния костной ткани вокруг имплантата, а также степени его остеоинтеграции. Для уточнения данных использовали прицельную рентгенограмму на радиовизиографе EzSensor (Vatech; Южная Корея). Некоторым пациентам перед операцией проводили компьютерную томографию на томограсре Brilliance 64 (Philips; Голландия).

Анализ состояния атрофии и качества костной ткани осуществляли в соответствии с основными классификациями типов костной ткани в зависимости от плотности и структуры челюстей по классификации Лехольма и Зарба [5]. Оценку состояния слизистой оболочки полости рта проводили, опираясь на классическую классификацию Суппле (табл. 1). Фиксировали наличие сопутствующих заболеваний и вредных привычек (курение).

Статистический анализ данных проводили с помощью программ Statistica версии 12, 2014 г. (StatSoft Inc., США) и MedCalc Statistical Software версии 18.2.1, 2018 г. (MedCalc Software bvba; Ostend, Бельгия). C их помощью оценивали факторы риска и шансы реализации с вычислением 95\%-го доверительного интервала. Выделенные переменные с высоким показателем шансов их реализации легли в основу первичного материала для многомерного статистического моделирования. Модели создавали в парадигме регрессионного оценивания, а именно процедурой логистической регрессии. Качество созданных моделей оценивали с помощью анализа характеристических кривых (receiver operator characteristic, или ROC-анализа).

\section{РЕЗУЛЬТАТЫ ИССЛЕДОВАНИЯ}

Первичный анализ показал, что характер осложнений, возникающих после установки временных конструкций на период остеоинтеграции имплантатов, довольно разнообразен. Частотное распределение случившихся осложнений в сформированных группах было следующим. Общее количество осложнений оказалось равным 29 (28\% от общего количества наблюдений). Наиболее распространенной (34\%) была тяжелая адаптация пациентов к полным съемным покрывным протезам, которую мы оценивали как осложнение. Следующими по частоте встречаемости стали мукозиты и переломы съемных покрывных протезов (по 20\% каждый). На третьем месте (с частотой по 10\%) были периимплантиты и аллергические реакции на мономер пластмассы, из которой изготавливали протезы. В 3\% случаев возникали галитоз и нарушение стабильности имплантата.

После того как было проведено сравнительное оценивание двух групп (первой, в которой протезирование прошло успешно, и второй, в которой у пациентов развились те или иные осложнения) по учитываемым параметрам, было сформировано представление о математическом разложении достоверно различающихся частот. Изучение риска развития осложнений у пациентов с полной вторичной адентией и потребностью во временном протезировании на момент остеоинтеграции установленных дентальных имплантатов проводили с оценкой вероятности того, что событие произойдет, в частности может развиться то или иное осложнение. Мы оценивали факторы риска как экспозицию, повышающую вероятность возникновения осложнения. Рассчитывали релятивистские риски как отношение частоты неблагоприятных исходов (факт развития осложнения) среди исследуемой группы, на вероятность развития которых оказывал влияние изучаемый фактор, к частоте возникновения осложнений среди исследуемых

таблица 1. Классификация слизистой оболочки протезного ложа по Суппле

\begin{tabular}{|l|l|l|}
\hline 1 класс & $\begin{array}{l}\text { Идеальный } \\
\text { рот }\end{array}$ & $\begin{array}{l}\text { Хорошо выраженные альвеолярные отростки, покрытые слегка податливой слизистой оболочкой, бледно-розового } \\
\text { цвета, без патологических процессов }\end{array}$ \\
\hline 2 класс & $\begin{array}{l}\text { Твердый } \\
\text { рот }\end{array}$ & $\begin{array}{l}\text { Атрофированная, плотная, сухая слизистая оболочка, места прикрепления складок несколько ближе к гребню } \\
\text { альвеолярного отростка, чем при 1 классе }\end{array}$ \\
\hline 3 класс & Мягкий рот & Гипертрофированная рыхлая слизистая оболочка, альвеолярные отростки низкие \\
\hline 4 класс & $\begin{array}{l}\text { Болтающийся } \\
\text { гребень }\end{array}$ & $\begin{array}{l}\text { Имеются подвижные тяжи слизистой оболочки, расположенные продольно и легко смещающиеся при } \\
\text { незначительном давлении отискной массы, тяжи могут быть ущемлены }\end{array}$ \\
\hline
\end{tabular}


в контрольной группе. Из всего набора переменных в результате статистического анализа осталось лишь шесть: степень атросии кости челюстей по классификации Лехольма и Зарба групп C, D, E; качество плотности кортикального и губчатого вещества кости класса III, IV по той же классификации; состояние слизистой оболочки полости рта типов 3, 4 по классификации Суппле; наличие аллергии на мономер пластмассы; плохое соблюдение гигиены полости рта и наличие вредных привычек (табл. 2).

Помимо этого, нами проведено изучение отношения шансов (ОШ). Шансы рассматривали как отношение вероятности того, что событие произойдет, к вероятности того, что событие не произойдет, или отношение вероятности действительного к вероятности недействительного, с целью оценки связи между фактом развития осложнения и фактором риска. При оценке ОШ установлено, что все выделенные ранее факторы риска имели достоверные вероятности реализации. Однако для нескольких факторов, а именно степени атросрии кости челюстей по классификации Лехольма и Зарба и состояния слизистой оболочки полости рта по классификации Суппле, шансы их реализации были неравнозначны. Так, степень атрофии была представлена тремя вероятностными состояниями, соответствующими пунктам C, D, E классификатора, а состояние слизистой - типам 3 и 4 [2, 6, 7] (табл. 3).

Учитывая полученные результаты, в оценке рисков при выборе вида временного протеза (ВВрП) на этапе остеоинтеграции имплантатов и шансов их реализации в различные этапы проводимого лечения, с целью повышения точности предсказательных оценок, было принято решение прибегнуть к процедуре многомерного статистического моделирования. С помощью логистической регрессии поставленная задача анализа связи между несколькими независимыми переменными и параметром отклика была успешно решена. Также было определено взаимное влияние признаков и вклад каждого на групповое

Таблица 2. Релятивистские риски возникновения осложнений при временном протезировании

\begin{tabular}{|l|c|c|c|}
\hline \multicolumn{1}{|c|}{ Фактор риска } & Релятивистский риск & "-" 95\% дИ \\
\hline Степень атрофии C, D, E & 1,7997 & 0,5518 & 0,4542 \\
\hline Качество кости III, IV & 0,9858 & 0,5566 & 2,1929 \\
\hline Состояние слизистой 3, & 1,3947 & 0,3044 & 2,2588 \\
\hline Аллергия + & 0,8716 & 0,2391 & 2,2069 \\
\hline Плохая гигиена + & 0,7891 & 0,2593 & 1,9864 \\
\hline Курение + & 0,5333 & 2,6781 \\
\hline
\end{tabular}

Таблица 3. Отношения шансов возникновения осложнений при временном протезировании

\begin{tabular}{|c|c|c|c|}
\hline Фактор риска & Отношение шансов & "-" 95\% ди & "+" 95\% ди \\
\hline Степень атрофии C & 1,8879 & 0,1518 & 2,2854 \\
\hline Степень атрофии D & 1,5858 & 0,1542 & 2,2546 \\
\hline Степень атрофии E & 1,2845 & 0,1566 & 2,2588 \\
\hline Состояние слизистой 3 & 1,2143 & 0,2044 & 2,2608 \\
\hline Состояние слизистой 4 & 1,1947 & 0,2721 & 1,9864 \\
\hline Аллергия + & 0,8333 & 0,2593 & 2,6781 \\
\hline Плохая гигиена + & 0,6891 & 0,2694 & 1,8871 \\
\hline Курение + & 0,4222 & 0,2443 & 2,5398 \\
\hline
\end{tabular}

Таблица 4. Характеристики регрессионной модели для ВВрП

\begin{tabular}{|l|c|}
\hline \multicolumn{1}{|c|}{ Показатели } & ВВрП \\
\hline Уровень значимости & $p=0,001$ \\
\hline Коэффициент детерминации Cox \& Snell R $R^{2}$ & 0,834 \\
\hline Коэффициент детерминации Nagelkerke $R^{2}$ & 0,758 \\
\hline Тест Ноsmer \& Lemeshow — значимость & $p=0,594$ \\
\hline Коэффициент конкордации & 0,8946 \\
\hline
\end{tabular}

Таблица 5. Стандартизованные регрессионные коэффициенты модели для ВВрП

\begin{tabular}{|l|c|c|c|}
\hline \multicolumn{1}{|c|}{ Переменная } & Коэффициент & Стандартная ошибка & Статистика Вальда \\
\hline Степень атрофии & $-2,13953$ & 0,40234 & 0,1203 \\
\hline Качество кости & 3,58284 & 0,83806 & 11,1382 \\
\hline Состояние слизистой & $-1,85714$ & 0,44173 & 11,6227 \\
\hline Аллергия & 3,46292 & 0,41697 & 10,968 \\
\hline Плохая гигиена & 1,55758 & 0,070499 & 0,6314 \\
\hline Курение & 0,056017 & 85,2702 & 0,01029 \\
\hline Constant & $-8,64908$ & & \\
\hline
\end{tabular}




\section{ORIGINAL RESEARCH I DENTISTRY}

разделение. Результаты моделирования представлены в табл. 4.

Созданная модель имеет высокую степень значимости. Значения обоих регрессионных коэфффициентов детерминации $\mathrm{R}^{2}$ оказались достаточно большими. Это позволяет предположить, что ее предсказательная устойчивость сравнительно высока. Подобное заключение также подтверждает вычисленный коэффициент конкордации. С помощью критерия Хосмера-Лемешова оценивали качество подгонки, сравнивая наблюдаемые частоты и расчетные. В нашем случае (хорошего согласия) имеем для этой статистики уровень значимости более 5\%. Стандартизованные регрессионные коэффициенты, включенные в модель, отражают все этапы, обозначенные

Таблица 6. Операционные характеристики модели для ВВрП в алгоритме. Ими стали переменные, представленные в табл. 5.

По результатам моделирования было составлено уравнение логистической регрессии, которое в общем виде выглядело следующим образом:

$$
\Phi=c+k_{1} x_{1}+k_{2} x_{2}+\ldots+k_{n} x_{n}
$$

где Ф - зависимая переменная; c - константа; $k_{j}-$ коэффициенты регрессионной функции; $x_{i}$ - предикторы, переменные.

С целью оценки построенной модели при проведении логистической регрессии и вычислении индивидуальных решений ее уравнения использовали графический

\begin{tabular}{|c|c|}
\hline Показатель & ВВрП \\
\hline Площадь под кривой ROC (AUC) & 0,921 \\
\hline Среднеквадратическая ошибка & 0,0524 \\
\hline$-95 \%$ ДИ (AUC) & 0,873 \\
\hline$+95 \%$ ДИ (AUC) & 0,963 \\
\hline$z$-статистика & 9,645 \\
\hline Уровень значимости $p$ (площадь $=0,5)$ & 0,001 \\
\hline Индекс Юдена J & 0,8284 \\
\hline Ассоциативный критерий & $\leq 1,43$ \\
\hline Чувствительность & 94,37 \\
\hline$-95 \%$ дИ (Se) & 76,2 \\
\hline$+95 \%$ ди (Se) & 98,7 \\
\hline Специфичность & 92,56 \\
\hline$-95 \%$ ДИ (Sp) & 79,8 \\
\hline +95\% дИ (Sp) & 97,6 \\
\hline +Отношение правдоподобия (+LR) & 7,31 \\
\hline$-95 \%$ ДИ (+LR) & 2,5 \\
\hline +95\% ДИ (+LR) & 21,4 \\
\hline -Отношение правдоподобия (-LR) & 0,18 \\
\hline$-95 \%$ ДИ (-LR) & 0,08 \\
\hline +95\% ДИ (-LR) & 0,4 \\
\hline +Прогностическая ценность (+PV) & 89,9 \\
\hline$-95 \%$ ДИ (+РV) & 75,3 \\
\hline +95\% ДИ (+РV) & 96,3 \\
\hline -Прогностическая ценность (-PV) & 82,2 \\
\hline$-95 \%$ ДИ (-PV) & 67,2 \\
\hline +95\% ДИ (-PV) & 91,3 \\
\hline
\end{tabular}

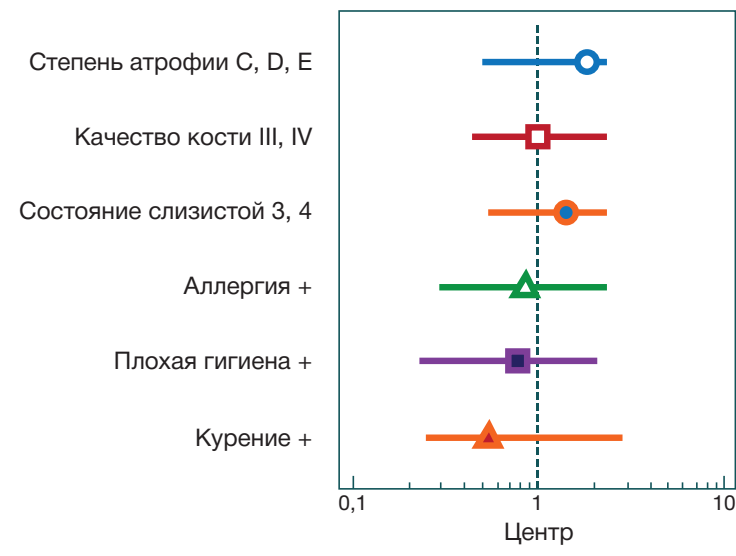

Рис. 1. Форест-диаграмма факторов риска вероятности осложнений при временном протезировании 


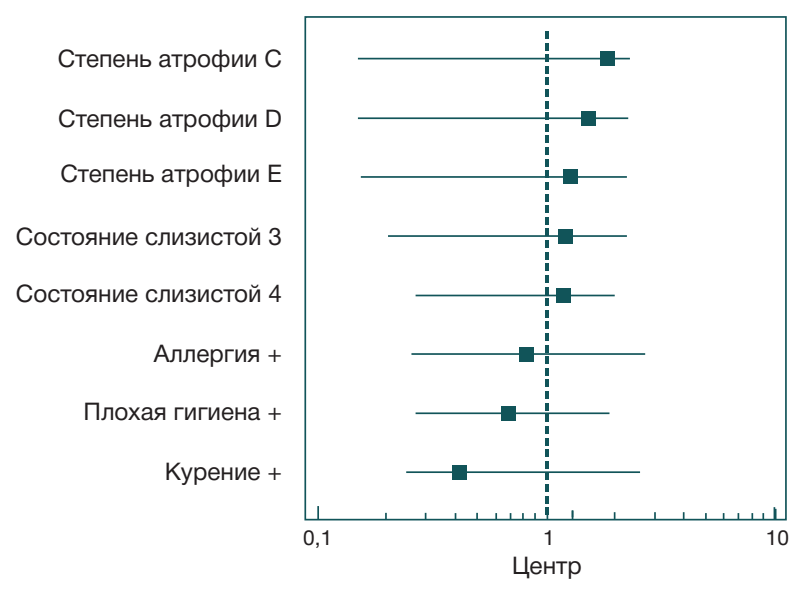

Рис. 2. Форест-диаграмма отношения шансов для факторов риска вероятности осложнений при временном протезировании

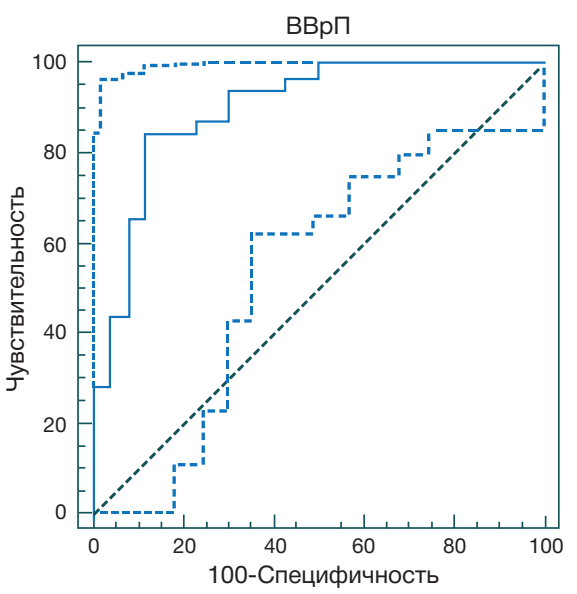

Pис. 3. ROC-кривая предсказательной модели для ВBрП

анализ и специальные формулы расчета оптимального значения величины порога отсечения - ROC-анализ. При этом вычисляли такие операционные характеристики метода, как площадь под кривой (AUC), индекс Юдена (Youden), ассоциативный критерий (optimal cut-off value), чувствительность и специфичность, положительное и отрицательное отношения правдоподобия (LR), положительные и отрицательные предиктивные уровни (PV) с определением 95\%-х доверительных интервалов для каждого показателя (табл. 6).

\section{ОБСУЖДЕНИЕ РЕЗУЛЬТАТОВ}

Из выделенных факторов риска ведущее место занимает качество плотности кортикального и губчатого вещества кости класса III, IV по классификации Лехольма и Зарба, что неудивительно, так как результат процедуры установки временного протеза напрямую зависит от этого. Далее следует наличие или отсутствие аллергии на мономер - от них зависит выбор материала, из которого изготавливают временный протез. Третьим по значимости фактором риска стала степень атрофии кости при оценке по классификации Лехольма и Зарба. Состояние слизистой оболочки полости рта 3-го и 4-го типов оказалось на четвертом месте. Помимо этого, факторами риска развития осложнений при проведении временного протезирования на момент остеоинтеграции установленных имплантатов оказались плохая гигиена полости рта и курение [2, 4]. На рис. 1 представлена форест-диаграмма распределения названных факторов риска.
При интерпретации результатов анализа ОШ реализоваться в осложнения при временном протезировании на момент остеоинтеграции дентальных имплантатов на беззубых челюстях в порядке убывания их вероятностей реализоваться представлены были следующие переменные: состояние слизистой оболочки 4-го типа по классификации Суппле, наличие аллергических реакций на мономер пластмассы протеза, состояние слизистой оболочки 3-го типа, степени атрофии кости челюстей E и D, плохая гигиена полости рта, степени атросии кости челюстей C и последнее место занимало курение. На рис. 2 представлена форест-диаграмма распределения названных ОШ.

Принимая во внимание полученные значения операционных характеристик модели, можно утверждать о применимости автоматизированного алгоритма для выбора способа временного протезирования. Цифры чувствительности и специфичности в верхних пределах их доверительных интервалов находятся на высоком уровне, при этом абсолютные значения так же высоки. Положительное отношение правдоподобия фактически в семь раз выше отрицательного, положительная прогностическая ценность также превышает отрицательную. Все это говорит об устойчивости модели в целом и подтверждается величиной площади под кривой, равной 0,921, представленной на рис. $3[8,9]$.

\section{ВЫВОДЫ}

1. Первым шагом в выборе оптимальной временной конструкции для реабилитации пациентов с полным отсутствием зубов с помощью покрывных съемных 
протезов или условно-съемных протезов на верхней и нижней челюстях с опорой на дентальные имплантаты на момент их остеоинтеграции является определение степени атрофии костей челюстей и плотности кортикального и губчатого вещества. 2. Непременным условием снижения риска возникновения осложнений является адекватность оценки состояния слизистой оболочки полости рта. 3. При плохой гигиене полости рта и курящему пациенту лучше предложить покрывной съемный протез. 4. При отсутствии аллергии на пластмассу и хорошей гигиене полости рта временные протезы могут быть изготовлены из любого вида пластмасс. 5. С учетом автоматизации поэтапного алгоритма может быть создана компьютерная программа для повышения степени объективизации в выборе способа временного протезирования и материала протеза.

\section{Литература}

1. McRory ME, Cagna DR. A technique for fabricating single screwretained implant-supported interim crowns in conjunction with implant surgery. J Prosthet Dent. 2014 Jun; 111 (6): 455-9. PubMed PMID: 24461941. DOI: 10.1016/j.prosdent.2013.11.005.

2. Aghaloo T, Pi-Anfruns J, Moshaverinia A, et al. The Effects of Systemic Diseases and Medications on Implant Osseointegration: A Systematic Review. Int J Oral Maxillofac Implants. 2019 Suppl; (34): 35-49. PubMed PMID: 31116832. DOI: 10.11607/ jomi.19suppl.g3.

3. Parvini P, Saminsky M, Stanner J, et al. Discomfort/pain due to periodontal and peri-implant probing with/without platform switching. Clin Oral Implants Res. 2019 Jul 20. PubMed PMID: 31325382. DOI: 10.1111/clr.13513.

4. Radzewski R, Osmola K. Osseointegration of Dental Implants in Organ Transplant Patients Undergoing Chronic Immunosuppressive Therapy. Implant Dent. 2019 Jul 12. PubMed PMID: 31306295DOI: 10.1097/ID.0000000000000916.

5. Lekholm U, Zarb G. Tissue-Integrated Prostheses Osseointegration in Clinical Dentistry. Chicago: Quintessence publishing, 1985; 199-210.

\section{References}

1. McRory ME, Cagna DR. A technique for fabricating single screwretained implant-supported interim crowns in conjunction with implant surgery. J Prosthet Dent. 2014 Jun; 111 (6): 455-9. PubMed PMID: 24461941. DOI: 10.1016/j.prosdent.2013.11.005.

2. Aghaloo T, Pi-Anfruns J, Moshaverinia A, et al. The Effects of Systemic Diseases and Medications on Implant Osseointegration: A Systematic Review. Int J Oral Maxillofac Implants. 2019 Suppl; (34): 35-49. PubMed PMID: 31116832. DOI: 10.11607/ jomi.19suppl.g3.

3. Parvini P, Saminsky M, Stanner J, et al. Discomfort/pain due to periodontal and peri-implant probing with/without platform switching. Clin Oral Implants Res. 2019 Jul 20. PubMed PMID: 31325382. DOI: 10.1111/clr.13513.

4. Radzewski R, Osmola K. Osseointegration of Dental Implants in Organ Transplant Patients Undergoing Chronic Immunosuppressive Therapy. Implant Dent. 2019 Jul 12. PubMed PMID: 31306295DOI: 10.1097/ID.0000000000000916.

5. Lekholm U, Zarb G. Tissue-Integrated Prostheses Osseointegration in

6. Hu Z, Wang X, Xia W, et al. Nano-Structure Designing Promotion Osseointegration of Hydroxyapatite Coated Ti-6Al-4V Alloy Implants in Diabetic Model. J Biomed Nanotechnol. 2019 Aug 1; 15 (8): 1701-13. PubMed PMID: 31219019. DOI: 10.1166/ jbn.2019.2812.

7. Mangano FG, lezzi G, Shibli JA, et al. Early bone formation around immediately loaded implants with nanostructured calciumincorporated and machined surface: a randomized, controlled histologic and histomorphometric study in the human posterior maxilla. Clin Oral Investig. 2017 Nov; 21 (8): 2603-11. PubMed PMID: 28154996. DOI: 10.1007/s00784-017-2061-y.

8. Широков И. Ю. Экспериментальное обоснование применения временных несъемных зубных протезов при дентальной имплантации [диссертация]. М., 2013.

9. Робакидзе Н. С., Лобановская А. А., Пекарчик Д. М. Применение временных протезных конструкций в период остеоинтеграции внутрикостных имплантатов. Институт стоматологии. 2016; (2): 78-9.

Clinical Dentistry. Chicago: Quintessence publishing, 1985; 199-210. 6. Hu Z, Wang X, Xia W, et al. Nano-Structure Designing Promotion Osseointegration of Hydroxyapatite Coated Ti-6Al-4V Alloy Implants in Diabetic Model. J Biomed Nanotechnol. 2019 Aug 1; 15 (8): 1701-13. PubMed PMID: 31219019. DOI: 10.1166/ jbn.2019.2812

7. Mangano FG, lezzi G, Shibli JA, et al. Early bone formation around immediately loaded implants with nanostructured calciumincorporated and machined surface: a randomized, controlled histologic and histomorphometric study in the human posterior maxilla. Clin Oral Investig. 2017 Nov; 21 (8): 2603-11. PubMed PMID: 28154996. DOI: 10.1007/s00784-017-2061-y.

8. Shirokov IYu. Jeksperimental'noe obosnovanie primenenija vremennyh nes\#emnyh zubnyh protezov pri dental'noj implantacii [dissertacija]. M., 2013.

9. Robakidze NS, Lobanovskaya AA, Pekarchik DM. Primenenie vremennyh proteznyh konstrukcij $\vee$ period osteointegracii vnutrikostnyh implantatov. Institut stomatologii. 2016; (2): 78-9. 